\title{
A pan-European survey of research in end-of-life cancer care
}

\author{
Katrin Ruth Sigurdardottir • Dagny Faksvåg Haugen • \\ Claudia Bausewein • Irene J. Higginson • \\ Richard Harding • Jan Henrik Rosland • Stein Kaasa • \\ on behalf of project PRISMA
}

Received: 18 August 2010 / Accepted: 11 November 2010/Published online: 30 November 2010

(C) The Author(s) 2010. This article is published with open access at Springerlink.com

\begin{abstract}
Background To date, there is no coordinated strategy for end-of-life (EOL) cancer care research in Europe. The PRISMA (Reflecting the Positive Diversities of European Priorities for Research and Measurement in End-of-life Care) project is aiming to develop a programme integrating research and measurement in EOL care. This survey aimed to map and describe present EOL cancer care research in Europe and to identify priorities and barriers.

Material and methods A questionnaire of 62 questions was developed and 201 researchers in 41 European countries were invited to complete it online in May 2009. An open invitation to participate was posted on the internet.

Results Invited contacts in 36 countries sent 127 replies; eight additional responses came through websites. A total of 127 responses were eligible for analysis. Respondents were 69 male and 58 female, mean age 49 (28-74) years; $85 \%$ of the scientific team leaders were physicians. Seventy-one of 127 research groups were located in a
\end{abstract}

Electronic supplementary material The online version of this article (doi:10.1007/s00520-010-1048-x) contains supplementary material, which is available to authorized users.

\author{
K. R. Sigurdardottir $(\varangle) \cdot$ D. F. Haugen $\cdot$ S. Kaasa \\ Department of Cancer Research and Molecular Medicine, \\ Faculty of Medicine, \\ Norwegian University of Science and Technology, \\ 7006 Trondheim, Norway \\ e-mail: katrin.sigurdardottir@haraldsplass.no \\ K. R. Sigurdardottir $\cdot$ J. H. Rosland \\ Sunniva Clinic for Palliative Care, \\ Haraldsplass Deaconess Hospital, \\ Bergen, Norway \\ K. R. Sigurdardottir · D. F. Haugen \\ Regional Centre of Excellence for Palliative Care, \\ Western Norway, Haukeland University Hospital, \\ Bergen, Norway
}

teaching hospital or cancer centre. Forty-five percent of the groups had only one to five members and $28 \%$ six to ten members. Sixty-three of 92 groups reported specific funding for EOL care research. Seventy-five percent of the groups had published papers in journals with impact factor $\leq 5$ in the last 3 years; $8 \%$ had published in journals with impact factor $>10$. Forty-four out of 90 groups reported at least one completed Ph.D. in the last 3 years. The most frequently reported active research areas were pain, assessment and measurement tools, and last days of life and quality of death. Very similar areas - last days of life and quality of death, pain, fatigue and cachexia, and assessment and measurement tools-were ranked as the most important research priorities. The most important research barriers were lack of funding, lack of time, and insufficient knowledge/expertise.

Conclusions Most research groups in EOL care are small. The few large groups (14\%) had almost half of the reported publications, and more than half of the current Ph.D.

C. Bausewein · I. J. Higginson · R. Harding Department of Palliative Care, Policy and Rehabilitation, Cicely Saunders Institute, King's College London,

London, UK

J. H. Rosland

Department of Surgical Sciences, Faculty of Medicine,

University of Bergen,

Bergen, Norway

S. Kaasa

Department of Oncology, St Olavs Hospital, Trondheim University Hospital,

Trondheim, Norway 
students. There is a lack of a common strategy and coordination in EOL cancer care research and a great need for international collaboration.

Keywords Neoplasms · Palliative care · Terminal care · Research · Europe $\cdot$ Survey

\section{Introduction}

More than 1.7 million people die from cancer in Europe each year, and the WHO predicts that the number will increase to over 2.1 million by 2020 (a 24\% increase) [1]. Estimates show that each year, 1.6 million cancer patients in Europe experience pain related to their disease, and one third of them will require complex treatment [2]. The number experiencing other distressing symptoms is also high [2]. Therefore palliative and end-of-life (EOL) care must be an essential component of cancer care and equally scientifically and evidence based. However, research in palliative care/ EOL care constitutes a very minor part of European cancer research. In the UK, less than $0.2 \%$ of research spending in cancer addresses palliative and EOL care [2]. Similar figures are not known for most other European countries, but are likely to be even lower for many of them.

Research in EOL care did not appear systematically on the agenda in Europe until the $1990 \mathrm{~s}$ with the establishment of the European Association for Palliative Care (EAPC) and its Research Network (EAPC-RN) in 1996 [3]. However, there is still little coordination of research in EOL cancer care in Europe today [4]. This may be due to lack of agreement on what constitutes EOL care, scarce information on public or clinical priorities, few appropriate measures of quality, and a lack of established best practice [5]. Methodological, organisational, attitudinal, and societal challenges have been identified, as well as a lack of research knowledge/skills in the area of EOL care research [6-9]. For the purposes of PRISMA, EOL care is defined as care in the last year of life.

In recent years, some well-funded palliative care research initiatives have emerged in Europe, Canada, the USA and Australia [10-13]. PRISMA is a project within the European Commission's Seventh Framework Programme with the overall purpose to deliver an integrated programme to co-ordinate research priorities, public priorities, and practice in EOL cancer care in Europe, with a focus on measurement [14]. In order to develop a future research agenda and strategic plan for EOL care in Europe, an overview of current research resources, activities, and challenges must be established. To our knowledge, no such overview currently exists. The National Cancer Research Institute's Strategic Planning Group on Supportive and Palliative Care report (SuPaC) [15] examined the past and present state of UK research in the field of supportive and palliative care for cancer, identified needs and opportunities for the future, clarified specific issues and barriers, and developed proposals for national strategic actions. An analysis of research workforce capacity and stakeholder views was in the form of a questionnaire survey. In addition to this survey from the UK, EOL care research has been surveyed in Latin America [16] and Australia [17]. The survey from Latin America (17 countries) aimed to determine the current status of palliative care research in Latin America. In order to develop a palliative research network in Queensland, Australia, a survey was conducted to map the current research activity, create a database and identify problems that had been encountered in undertaking research [17].

The aim of the present study was to conduct a questionnaire survey to map EOL cancer care research across Europe and to identify priorities, needs and barriers in this research area.

\section{Material and methods}

\section{Questionnaire}

A cross-sectional online self-completed survey comprising 62 items was developed in English (Electronic Supplementary Material). The survey focused on clinical research in adults with cancer in their last year of life. Clinical research was defined as research directly involving patients or family members/care givers. The questions addressed research conducted in the previous 3 years, current ongoing research, and future plans. In addition to demographic characteristics, questions were related to research topics, methodology and trial design, staffing, organisation and funding, quality indicators (completed doctoral degrees in the last 3 years, number of publications in peer-reviewed journals last 3 years, number of conference abstracts last 3 years), and priorities in, and barriers to, research.

An outline of the contents of the questionnaire was developed at the first meeting of PRISMA work package 3 (WP3). Based on this draft and consulting identified related surveys [15-17], two WP3 members selected and designed questions. The draft was circulated to all WP3 members and PRISMA work package leads for comments. Some of the items were adapted from the SuPaC survey [15]. The list of EOL care research topics was based on the abstract categories used for the EAPC conferences and the list of research topics used in the SuPaC survey [3, 15].

A web-based solution was developed using SelectSurveyASP Advanced 8.1.10 software [18]. It was pilot tested by nine experts in EOL care. Respondent access level was single response. Respondents returning a completed survey could edit their original responses. The survey was 
designed with a combination of mandatory and optional responses. Skip logic was used.

\section{Sample}

We wanted to include all chairs in palliative medicine, palliative care or EOL care in Europe. In countries without such chairs, palliative care researchers or other identified contact persons were approached. Through the EAPC, Open Society Institute, UK Hospice Information Service, leaders of national associations for palliative care and/or palliative medicine in European countries, literature searches, and a number of international contacts, a list of 233 contacts in a total of 41 European countries was identified. At the country level, e-mails were sent with information about the survey and lists of proposed respondents in each country, asking about chairs in palliative medicine and also if important researchers were missing from our list. Contacts in 28 countries responded to our inquieries. By January 2009, a list of 201 chairs, researchers or other contact persons in 41 European countries had been compiled. All of these received a personal invitation to participate by e-mail with a link to the survey. In addition, an open invitation to participate was posted on the websites of the EAPC [3] and the European Palliative Care Research Collaborative [11].

The data collection started on the 30th of April 2009 and closed after 1 month. Reminders were sent to nonresponders after 2 and 3 weeks.

\section{Analyses}

The data was cleaned and checked to identify and correct potential divergence/errors and ensure missing data was coded uniformly. The responses were entered into an SPSS database version 15.0. Data was analyzed using descriptive statistics.

We hypothesised that the state of EOL care research in a country would be linked to the development of palliative care in that country. Due to the limited number of responses from many countries it would be difficult to compare countries or even regions within Europe. We decided to divide the countries into two groups based on their level of palliative care development, as described by the International Observatory on End-of-Life Care in 2008 [19]. The following categorisation was developed through a multimethod approach combining published and grey literature, information from websites, directories, and databases, expert opinion, and the work of a task force of the European Association for Palliative Care [19]:

1. Countries with developed palliative care (i.e. countries where palliative care activities are approaching integra- tion with mainstream service providers): Austria, Belgium, Denmark, Finland, France, Germany, Hungary, Iceland, Ireland, Italy, Netherlands, Norway, Poland, Romania, Slovenia, Spain, Sweden, Switzerland, and UK.

2. Countries with underdeveloped palliative care (i.e., countries with localised palliative care provision only): Belarus, Bosnia and Herzegovina, Bulgaria, Croatia, Czech Republic, Estonia, Georgia, Greece, Latvia, Lithuania, Luxembourg, Moldova, Montenegro (no known activity), Portugal, Russia, Serbia, and Ukraine.

Ethics

We contacted the Regional Committee for Medical Research Ethics in Central Norway. Due to the nature of the study no application was required.

\section{Results}

\section{Responses}

We received 127 responses from the 201 invited contacts. The responses came from 36 European countries (Table 1). A further eight replies were submitted in response to the open invitation on the websites. Eight responses were excluded, three duplicates and five non-serious responses submitted through the websites.

Altogether, 127 responses were eligible for analysis: 104 complete (all mandatory questions answered) and 23 incomplete responses. Respondents were 69 (54.3\%) male and $58(45.7 \%)$ female, mean age of 49.4 (range, 28-74) years.

Location of research groups

Seventy-one of the 127 groups were located in either a teaching hospital (44) or a cancer centre (27), with a further 18 being on a university campus but not attached to a hospital. Ten groups were based in hospices and eight in district general hospitals.

\section{Work force}

The survey showed that $85 \%$ of the scientific team leaders were physicians (104/123), most commonly with a palliative medicine background, followed by oncology.

The survey identified a total of 965 research staff in 88 groups. The mean number of members in a research group was $11(1-58) ; 460(48 \%)$ had part-time and $505(52 \%)$ full-time positions. Eighty-three groups reported a total of 
Table 1 Number of responses per country

371 research staff having combined positions with clinical work.

Ninety-two groups reported on their research staff. Seventy-three $(79 \%)$ of these groups had scientific team leaders who were professors or held another academic position (95 team leaders in all), 67 groups had a total of 128 experienced/senior researchers, and 52 groups reported having a total of 119 early researchers or post-docs. There were $172 \mathrm{Ph} . \mathrm{D}$. students in 54 groups, and 32 groups had a total of 111 Master degree students. More than half of the current Ph.D. students are in the few large groups. Thirty groups reported having managers/administrators, and 33 groups reported a total head count of 59 technical staff.
Most of the groups were small: $45 \%$ of the groups had one to five members, and $28 \%$ six to ten members. Thirteen groups (14\%) were defined as large groups, having more than 15 members.

Thirty-nine of the groups were multidisciplinary, defined as having four or more different disciplines represented among the research staff. A wide range of professions contribute to the research workforce, including physicians $(35 \%)$, nurses $(29 \%)$, psychologists $(8 \%)$ and sociologists (4\%) (Fig. 1).

Research network, collaboration and strategy

National collaboration with other palliative care research groups was reported by $77 / 92$ groups (84\%); 60/92 (65\%) reported international research collaboration.

Four out of 33 countries (12\%) reported to have a formalised palliative care research network (Estonia, France, Switzerland and Ukraine; Estonia and Ukraine had only one respondent each). Twelve countries answered this question with both "yes" and "no". Twenty-four of 33 countries (73\%) reported no national strategy for EOL care research; from eight countries (24\%) there were both "yes" and "no" answers to this question. One country reported having a national strategy for EOL care research; however, this country had only one respondent.

Funding

Seventy-four of the 92 groups ( $80 \%$ ) answered the question concerning funding sources; 63 (85\%) of these had specific funding for research on EOL care. Out of this number, 60 were groups in countries with developed palliative care services. The funding sources were governmental (76\%), institutional (45\%), pharmaceutical $(45 \%)$, charity $(42 \%)$, international $(33 \%)$, scientific awards (30\%), and private (16\%).

Research completed in the last 3 years

One hundred and four $(86 \%)$ out of 121 research groups reported completed research on EOL care in the previous 3 years. Ninety-six groups $(92 \%)$ reported on their specific areas of research and the most frequently reported areas were pain (82\%), assessment and measurement tools (69\%), last days of life and quality of death (56\%), other symptoms $(55 \%)$, and policy and the organisation of services $(53 \%)$.

A total of 1,714 (median, 11; range, 0-149) conference abstracts were published from 89 research groups in the last 3 years.

The total number of publications in peer-reviewed journals in the last 3 years was 1,329 (median, 7; range 0-154) from the 89 groups. Nine groups had no publications. Five percent of the publications were from countries with underdeveloped 
Fig. 1 Discipline of the research staff in 88 groups. Numbers working full time and part time. Note that physicians are spread over several categories

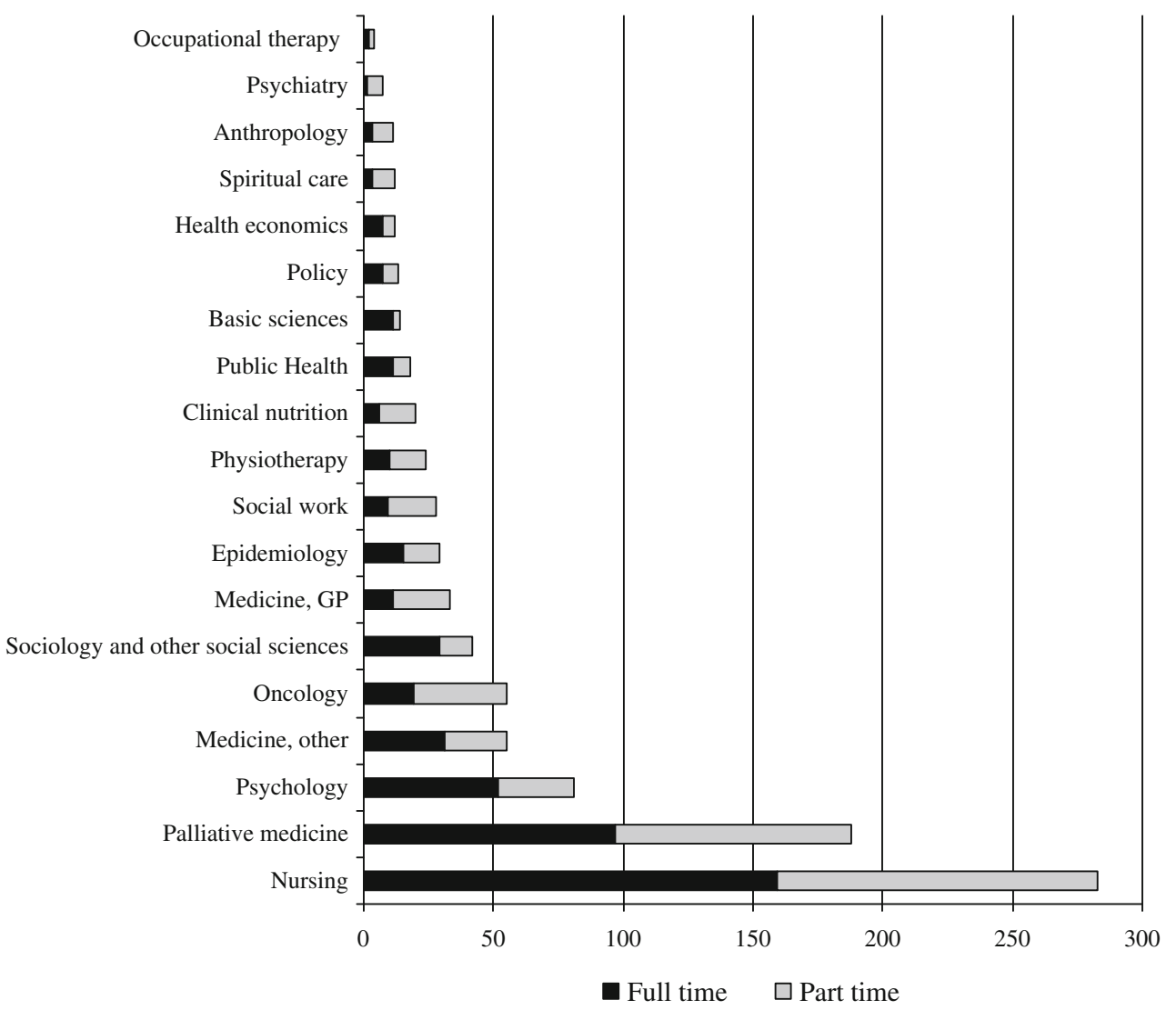

the research was conducted in countries with developed palliative care services (16 countries). In 90/95 groups $(95 \%)$ the research was directly involving patients, and in $54 / 95$ (57\%) directly involving family members.

Figure 2 reports the research topics, reported by 94 groups. The most frequently reported areas of research were pain $(70 \%)$, assessment and measurement tools $(63 \%)$, other symptoms (52\%), last days of life and quality of death (44\%), and family and caregivers (40\%).

The research methodologies reported by the groups $(n=89)$ were clinical trials $(74 \%)$, audit and quality control (51\%), health service research (38\%), production of guidelines (34\%), systematic reviews and meta-analysis (31\%), epidemiology $(30 \%)$, basic research $(15 \%)$ and translational research $(12 \%)$.

The study designs of the clinical trials $(n=66)$ were randomised controlled trials (65\%), observational studies $(61 \%)$, prospective nonrandomized trials $(58 \%)$, qualitative studies $(47 \%)$, retrospective studies (35\%), and cross-sectional studies $(32 \%)$.

\section{Barriers to EOL care research}

Figure 3 presents barriers in general $(n=104)$. More than half of the groups considered the following barriers of importance: lack of funding (85\%), lack of time (64\%), insufficient knowledge and expertise (62\%), lack of personnel (54\%), and lack of infrastructure and support (52\%). Groups in research at the time of the survey. Eighty-four percent of 
Fig. 2 Ongoing research. Research topics. Number of groups

Fig. 3 Barriers in general. Number of groups
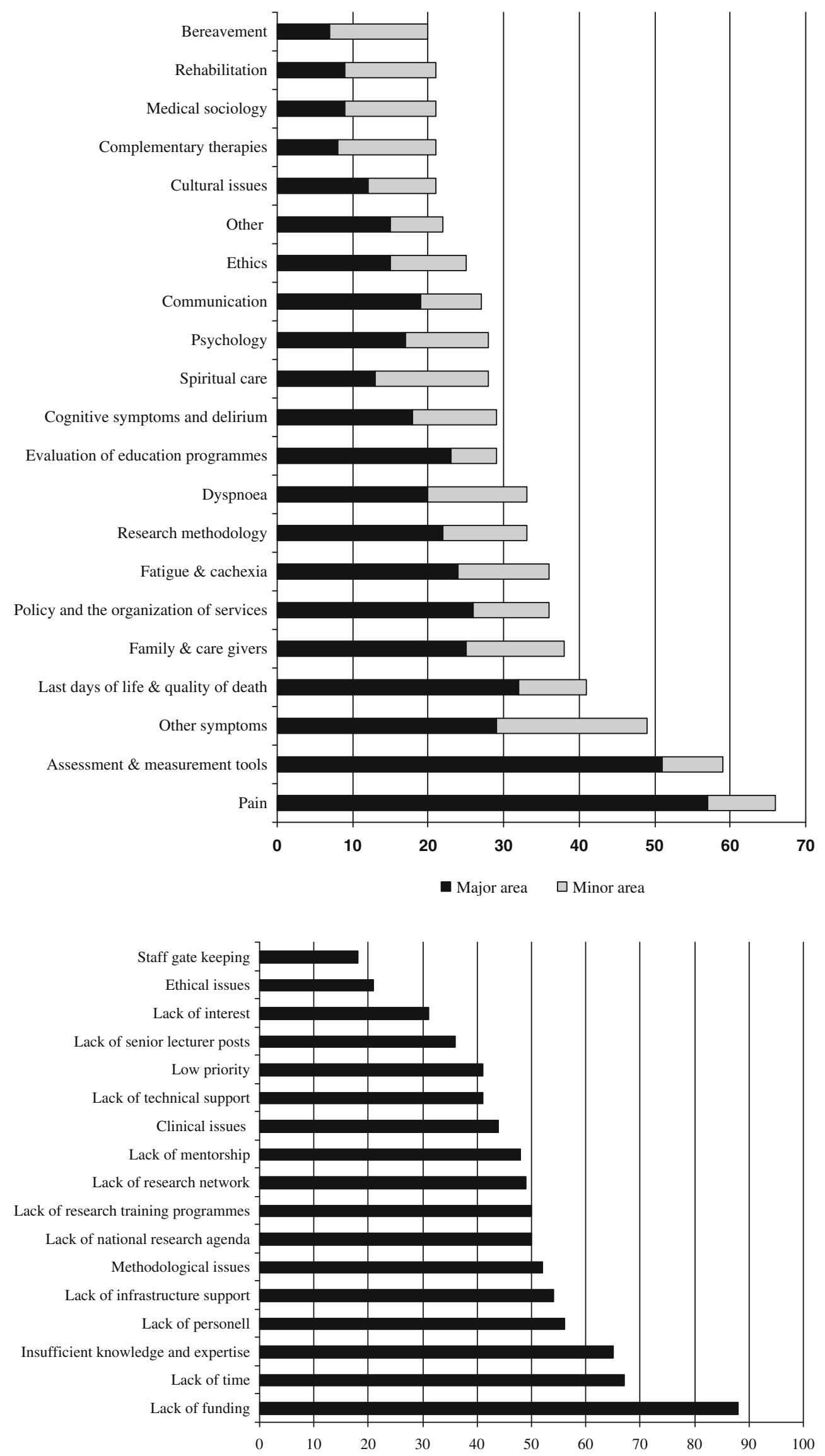
countries with underdeveloped palliative care services reported more lack of technical support (52\% vs 35\%), lack of research training programmes (67\% vs $42 \%)$, and lack of a national research agenda (63\% vs $43 \%$ ). On the other hand, countries with developed palliative care services reported more often clinical issues (51\% vs $18 \%$ ), lack of time $(69 \%$ vs $52 \%)$, and staff gate keeping (21\% vs $7 \%)$ as barriers.

Priorities for the field

Figure 4 presents the future research priorities for the field $(n=104)$. More than half of the groups reported that research on last days of life and quality of death $(63 \%)$, pain (61\%), fatigue and cachexia (60\%), assessment and measurement tools (59\%), cognitive symptoms and delirium $(56 \%)$, policy and the organisation of services $(54 \%)$, and communication (51\%) should be prioritised.

\section{Discussion}

To our knowledge, this is the first study reporting on the current state of EOL cancer care research across Europe.
The most frequently reported current research areas were pain, assessment and measurement tools, other symptoms, and last days of life and quality of death. The results show that although some large groups were identified, most of the research groups were small. Almost 50\% of all positions were part time. Very few European countries reported to have a formalised palliative care research network. Lack of funding was the most commonly reported barrier.

\section{Workforce}

A previous, limited survey of palliative care research in 13 European countries suggested that there were very few groups reaching a critical size [21]. This impression is confirmed by the present study. Even though a few large groups exist, the picture is dominated by small and scattered groups and initiatives, without national or international coordination. The few large groups reported almost half of the publications, and more than half of the current $\mathrm{Ph} . \mathrm{D}$. students are in these groups. These findings are also well in line with the $\mathrm{SuPaC}$ report [15], which also found that the palliative care research workforce was fragmented
Fig. 4 Priorities for the field concerning future research on adults with cancer in their last year of life. Number of groups

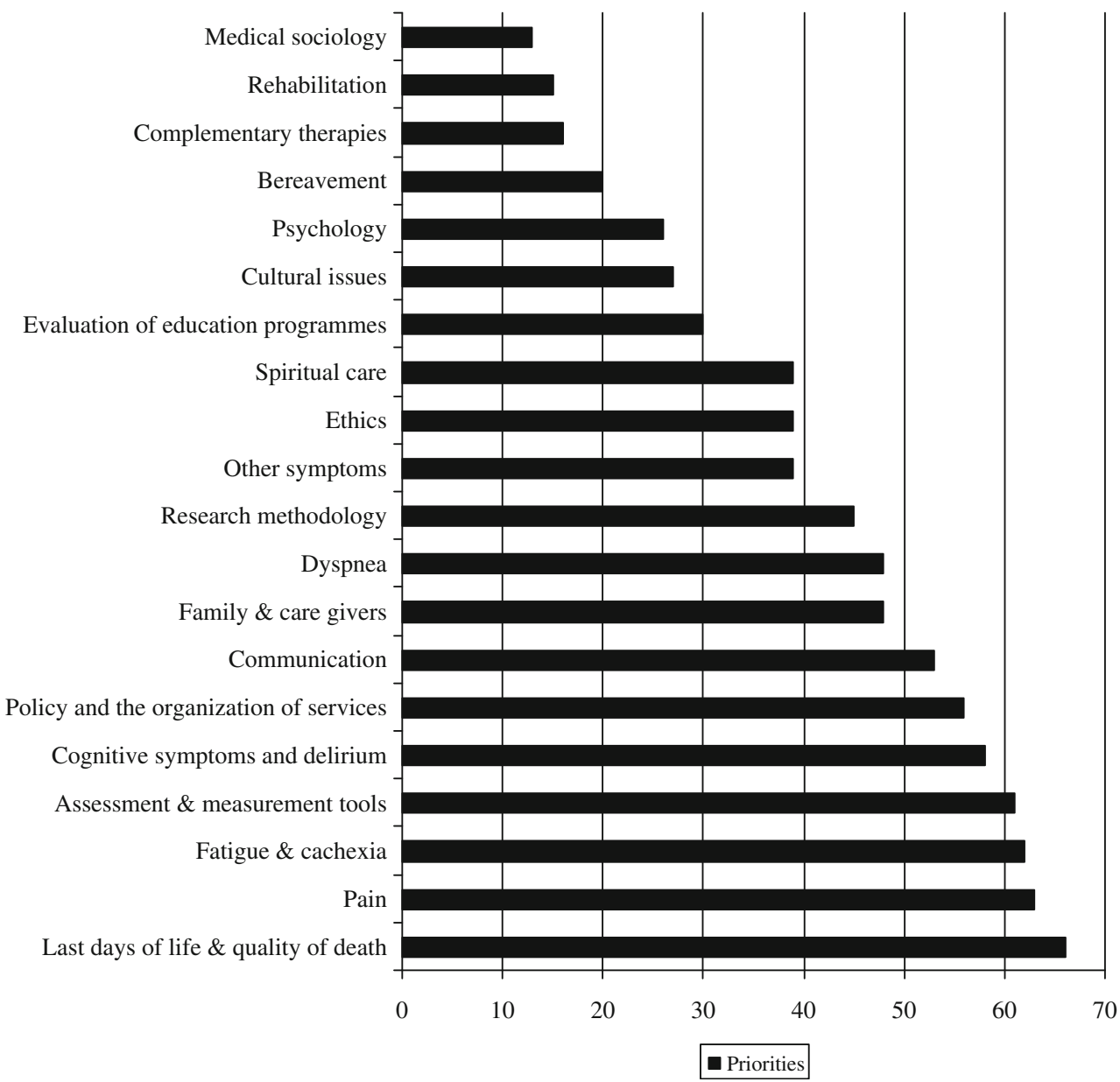


across multiple sites; there were inadequate numbers of postdoctoral and senior lecturers, and scarce infrastructure, with poor access to expertise in research methodology.

Our findings show a great need for structured collaboration both nationally and internationally to promote EOL cancer care research in Europe. Larger groups should try to include smaller groups in their work. Studies have shown that research groups are complex interacting systems with a strong size dependency, and that is it important to achieve critical mass [22].

Research topics and methodology

The most frequently reported areas of completed research were pain, assessment and measurement tools, last days of life and quality of death, other symptoms, and policy and the organisation of services. Ongoing research showed the same areas, but less research on policy and organisation, and more on family and caregivers. All these areas were included in the list of the most common research topics in the $\mathrm{SuPaC}$ report of 2004 [15]; however, the most commonly reported area of research in the SuPaC survey was psychological research, followed by information/ communication. While the $\mathrm{SuPaC}$ report was restricted to the UK, the present survey had respondents from 36 European countries.

The strong focus on symptomatology may reflect the predominantly medical sample in our survey. However, symptom relief is at the core of palliative care, and a review of palliative care research publications by Kaasa and coworkers in 2006 found that symptom control/management was one of the two most frequent topic areas, service development (policy and organisation) being the other [21]. The literature review [21] indicated that the majority of research publications in palliative care were surveys and descriptional/observational studies, and that rather few randomised, controlled trials were published. These findings were confirmed in 2008 in a paper on research methodologies in palliative care [23]. In the present survey, 66 out of 89 groups reported conducting clinical trials, and the most common study design for the clinical trials was the randomised controlled trial ( $65 \%$ of the groups), followed by observational studies (61\%) and prospective nonrandomized trials (58\%). Randomised, controlled trials remain the gold standard for evidence, and a development with more groups doing this type of studies would be very welcome. However, it remains to be seen whether this finding will be reflected in publications over the next years.

\section{Barriers}

More than half of the groups reported lack of funding, lack of time, insufficient knowledge and expertise, lack of personnel and lack of infrastructure support as barriers to EOL care research. Similar barriers have been identified by others [15-17, 24]. Almost one third of the responders in the present study had no specific funding for EOL care research. Sixty-three groups had specific funding for research on EOL care and 60 of these were groups in countries with developed palliative care services. Thus the state of palliative care research is closely linked to the development of clinical palliative care services in a country. Research collaboration between countries with developed palliative care services and countries with less developed palliative care could also enhance the development of clinical palliative care services in the latter. Ear-marked funding to boost the efforts can be crucial in these countries.

Our data shows that almost half of the research staff (48\%) had combined clinical and research posts. People in combined positions often face serious difficulties in relation to pressure from clinical commitments, and this might result in lack of time being ranked as the second most important barrier. This point is important to address when planning combined clinical and academic careers.

\section{Priorities}

A number of research topics were identified as priority areas for the future (Fig. 4). The future priority areas identified by the respondents were very similar to the areas in which the respondents were currently working (Fig. 2), except that research into the last days of life and quality of death emerged as a stronger priority. It is possible that the respondents often prioritised areas of research with which they were already familiar. However, several of the priority topics from the present survey overlap with the ten areas identified with urgent need for increased research efforts at the Birmingham International Workshop on Supportive, Palliative, and End-oflife Care Research in 2005 [25]. The Birmingham group also included symptomatology in their priority areas: measuring symptoms and symptoms clusters, study of the biological bases of complex symptom clusters and enhancing research in symptom interventions. The priority areas identified in the present survey have later been further developed in an international workshop with 30 participants from 25 European countries selected from the survey responders [26]. These priorities, as defined by clinicians and researchers, will later be integrated with public priorities and preferences in the context of PRISMA and give guidance to coordinating bodies aiming to promote pan-European research [14].

\section{Study limitations}

The present study is the first broad survey of EOL care research in Europe. The invitation to participate in the survey was sent to all chairs (professors) in EOL care, 
palliative care, and palliative medicine in Europe, and to other leaders of research groups in these areas that we were able to identify. Despite our broad approach to identify professionals involved in relevant research, we might have missed groups, especially research groups dealing with EOL care issues in areas defined outside EOL care or palliative care.

Although the survey had a high response rate $(67 \%)$, there might be a selection bias among the respondents. The majority of responders belonged to countries with developed palliative care (76\%). However, this is where most EOL care research groups are located. The majority of the scientific team leaders in the responding groups were physicians. This predominance has probably led to more biomedically oriented answers than if researchers from other professions had been asked about their priorities.

The language, format and contents of the survey questionnaire, especially the proffered lists of response options, represent additional limitations. One example is the list of EOL care research topics, which also was used for defining future needs and priorities.

\section{Conclusions}

The results of this pan-European survey demonstrate that EOL cancer care research is in its infancy. Small and scattered groups and initiatives without national or international coordination dominate the picture. However, a few large groups exist. These large groups reported almost half of the publications, and more than half of today's Ph.D. students are in these groups.

We conclude that there is a great need for structured collaboration both nationally and internationally to promote palliative and EOL cancer care research in Europe. The EAPC-RN, the European Palliative Care Research Centre [27] and Cicely Saunders Institute [28] all have important roles in facilitating future EOL care research and organising collaborative research efforts. Hopefully this will facilitate high-quality research within Europe and enhance the development of clinical palliative care.

Acknowledgement We would like to thank the Open Society Institute and other institutions and individuals who helped us identify our sample, and Berit Bjelkåsen for technical assistance. We also thank all the researchers who took part in the survey. PRISMA, Reflecting the Positive Diversities of European Priorities for Research and Measurement in End-of-Life Care, is funded by the European Commission's Seventh Framework Programme (contract number: Health-F2-2008-201655) with the overall aim to co-ordinate highquality international research into end-of-life cancer care. PRISMA aims to provide evidence and guidance on best practice to ensure that research can measure and improve outcomes for patients and families. PRISMA activities aim to reflect the preferences and cultural diversities of citizens, the clinical priorities of clinicians, and appropriately measure multidimensional outcomes across settings where end-of-life care is delivered. Principal Investigator: Richard Harding. Scientific Director: Irene J Higginson. Work Package 3 Lead: Stein Kaasa. PRISMA members: Gwenda Albers, Barbara Antunes, Ana Barros Pinto, Claudia Bausewein, Dorothee BechingerEnglish, Hamid Benalia, Lucy Bradley, Lucas Ceulemans, Barbara A Daveson, Luc Deliens, Noël Derycke, Martine de Vlieger, Let Dillen, Julia Downing, Michael Echteld, Natalie Evans, Dagny Faksvåg Haugen, Lindsay Flood, Nancy Gikaara, Barbara Gomes, Marjolein Gysels, Sue Hall, Richard Harding, Irene J Higginson, Stein Kaasa, Jonathan Koffman, Pedro Lopes Ferreira, Johan Menten, Natalia Monteiro Calanzani, Fliss Murtagh, Bregje Onwuteaka-Philipsen, Roeline Pasman, Francesca Pettenati, Robert Pool, Tony Powell, Miel Ribbe, Katrin Sigurdardottir, Steffen Simon, Franco Toscani, Bart van den Eynden, Jenny van der Steen, Paul Vanden Berghe, Trudie van Iersel.

Conflicts of interest statement None declared.

Open Access This article is distributed under the terms of the Creative Commons Attribution Noncommercial License which permits any noncommercial use, distribution, and reproduction in any medium, provided the original author(s) and source are credited.

\section{References}

1. World Health Organization/UICC (2003) Global action against cancer. World Health Organization/UICC, Geneva

2. Higginson IJ, Costantini M (2008) Dying with cancer, living well with advanced cancer. Eur J Cancer 44:1414-1424

3. The European Association for Palliative Care Research Network (EAPC RN). Available at: http://www.eapcnet.org/. Accessed 25 June 2010

4. Kaasa S, Radbruch L (2008) Palliative care research - priorities and the way forward. Eur J Cancer 44:1175-1179

5. Shipman C, Gysels M, White P, Worth A, Murray SA, Barclay S, Forrest S, Shepherd J, Dale J, Dewar S, Peters M, White S, Richardson A, Lorenz K, Koffman J, Higginson IJ (2008) Improving generalist end of life care: national consultation with practitioners, commissioners, academics, and service user groups. BMJ 337:848-851

6. Palliative Medicine (2006) Special issue on palliative care research methodology. Palliat Med 20:725-871

7. Christakis NA (2006) Advances in palliative care research methodology. Palliat Med 20:725-726

8. Kaasa S, De Conno F (2001) Palliative care research. Eur J Cancer 37:153-159

9. Patrick DL, Ferketich SL, Frame PS, Harris JJ, Hendricks CB, Levin B, Link MP, Lustig C, McLaughlin J, Ried LD, Turrisi AT 3 rd, Unutzer J, Vernon SW (2003) National Institutes of Health state-of-the-science conference statement: symptom management in cancer: pain, depression, and fatigue, July 15-17, 2002. J Natl Cancer Inst 95:1110-1117

10. OPCARE9. Available at: http://www.opcare9.eu/. Accessed 25 June 2010

11. European Palliative Research Collaborative. Available at: http:// www.epcrc.org/. Accessed 25 June 2010

12. Fainsinger RL (2008) Global warming in the palliative care research environment: adapting to change. Palliat Med 22:328-335

13. Defining best practice in palliative care in Europe. Available at: http://www.painandpalliativemedicine.eu/. Accessed 25 June 2010

14. Harding R, Higginson IJ (2010) PRISMA: a pan-European coordinating action to advance the science in end-of-life cancer care. Eur J Cancer 46:1493-1501 
15. National Cancer Research Institute (NCRI) (2004) Supportive and palliative care research in the UK: report of the NCRI Strategic Planning Group on Supportive and Palliative Care. NCRI, London. pp. 1-64.

16. Wenk R, De Lima L, Eisenchlas J (2008) Palliative care research in Latin America: results of a survey within the scope of the Declaration of Venice. J Palliat Med 11:717-722

17. Hardy J, Dingle K, Beebe H, Yates P (2005) Creating a palliative research network in Queensland, Australia - is this the answer? Prog Palliat Care 13:277-281

18. SelectSurveyASP Advanced 8.1.10 software. Available at: http:// www.classapps.com/. Accessed 25 June 2010

19. Wright M, Wood J, Lynch T, Clark D (2008) Mapping levels of palliative care development: a global view. J Pain Symptom Manage 35:469-485

20. Web of Knowledge-Science-Thomson Reuters. Available at: http://wokinfo.com/. Accessed 25 June 2010

21. Kaasa S, Hjermstad MJ, Loge JH (2006) Methodological and structural challenges in palliative care research: how have we fared in the last decades? Palliat Med 20:727-734
22. Kenna R, Berche B (2010) Critical mass and the dependency of research quality on group size. pp. 1-15. Available at: http://arxiv.org/

23. Payne SA, Turner JM (2008) Research methodologies in palliative care: a bibliometric analysis. Palliat Med 22:336-342

24. Binswanger J, Inauen R, Strasser F (2006) Barriers to Palliative Care (PC) research. Palliat Med 20: abstr \# 467

25. Hagen NA, Addington-Hall J, Sharpe M, Richardson A, Cleeland CS (2006) The Birmingham International Workshop on supportive, palliative, and end-of-life care research. Cancer 107:874-881

26. Sigurdardottir KR, Haugen DF, van der Rijt CC, Sjogren P, Harding R, Higginson IJ, Kaasa S (2010) Clinical priorities, barriers and solutions in end-of-life cancer care research across Europe. Report from a workshop. Eur J Cancer 46:18151822

27. European Palliative Care Research Centre. Available at: http:// www.ntnu.edu/prc. Accessed 25 June 2010

28. Cicely Saunders Institute. Available at: http://www.cicelysaun dersfoundation.org/cicely-saunders-institute. Accessed 25 June 2010 